\title{
Measuring unbalance-induced vibrations in rotating tools
}

\author{
Martin Kimmelmann ${ }^{1, *}$, and Thomas Stehle ${ }^{1}$ \\ ${ }^{1}$ Institute for Machine Tools, University of Stuttgart, 70174 Stuttgart, Germany
}

\begin{abstract}
Unbalances in a tool cause vibrations of the spindle and the machine itself and lead to a waviness of the machined workpiece surface. This paper presents an experimental and analytical procedure for optically measuring the unbalance-induced displacements of the tool centre point (TCP). Therefore, a new method is introduced to determine the dynamic vibrations of a tool by comparing the geometrical profile of the tool with the dynamical profile at a high rotational speed. The necessary steps for measuring the signals and calculating the underlying dynamic vibrations of the tool are presented here. Afterwards, the unbalance-induced vibrations of a milling tool are shown as well as their influence on the eccentricity of the rotation axis. With this newly introduced method it is possible to directly link the displacements of the tool under rotation to the waviness of the workpiece surface and the dynamic stiffness of machine tools.
\end{abstract}

\section{Introduction}

Unbalance in rotating components generally leads to a deviation of the shaft axis from the axis of rotation [1]. Rotating tools such as drills and milling cutters produce centrifugal forces depending on rotational speed, which can cause the tools themselves or the machine to vibrate. In order to keep these vibration effects caused by unbalance, especially in the bearing planes and the tool centre point, within tolerable limits, the tools must often be balanced [2]. The vibrations separately excited by unbalance lead to dynamic displacements of the tool, which may show on the workpiece surface [3]. In particular in the machining of wood and derived wood products, the tool's condition of unbalance plays an important part owing to the generally high cutting speeds. When unbalance causes great eccentricities here, it may happen that only one cutting edge of the tool produces the workpiece surface determining the quality. Although the other cutting edges are involved in the cutting process as well, only the cutting edge protruding furthest, however, produces the workpiece surface in this so-called single-edged finishing [4]. Concerning high-quality and productive machining processes, such as precision planing, it is therefore necessary to equalise the centre point path radii of the cutting edges by the so-called jointing method at operating speed in order to attain the desired workpiece quality [5].

Unbalance in tools is limited according to two points of view: the bearing loads caused by unbalance and the guarantee of tool safety. In DIN 69888, based on a simplified model

${ }^{*}$ Corresponding author: martin.kimmelmann@,ifw.uni-stuttgart.de 
for motor spindles, the load on the front bearing point caused by unbalance is limited to $1 \%$ of the dynamic basic load rating $C_{d y n}$ of the bearing [6]. The requirements for balancing are laid down in ISO 15641 and EN 847 regarding high circumferential speeds on tools, typical of wood machining processes $[7,8]$. The influence of unbalance on the machining quality and the tool wear in wood machining is being analysed in the IGF project $18381 \mathrm{~N}$ „Establishing the necessary balance quality of tools in wood machining", funded by the Federal Ministry for Economic Affairs and Energy (BMWi). The goal here is to establish economically sensible, required balance qualities for wood machining.

\section{Procedure for identifying vibrations in the TCP}

Unbalances in tools are centrifugal forces due to the rotation of the tool. According to Equation (1), the unbalance $U$ here causes the centrifugal forces $F_{U}$, which are quadratic to the angular velocity $\omega$. In practice, unbalance is mostly given as the product of the unbalance mass $u$ and the eccentricity of the centre of mass $e$ as well [1]. The unit of unbalance is usually given in gmm here.

$$
F_{U}=U \cdot \omega^{2}=u \cdot e \cdot \omega^{2}
$$

Unbalances here work as preloading forces on the tool depending on rotational speed. Due to these forces, the tool axis is eccentrically displaced in the direction of the radial force. In this way, the tool axis moves around the axis of rotation with an eccentricity e. In order to compare different kinds of rotors with each other, the balance quality $G$ was introduced as parameter in ISO 1940 [9] and describes the circumferential speed of the tool axis around the axis of rotation, cf. Equation (2).

$$
G=e \cdot \omega
$$

As an example, figure 1 illustrates a circle rotating centrically as well as one rotating eccentrically around the eccentricity $e$. In the stationary coordinate system, a constant distance signal was recorded in the case of the centrically rotating circle and a signal depending on the angle $\varphi$ and the eccentricity $e$ was recorded in the case of the eccentrically rotating circle. The centre point curve of the eccentrically rotating circle in the $\mathrm{X}$ - and $\mathrm{Y}$ directions can be calculated from the difference between the distance signals, according to Equations (3) und (4):

$$
\begin{aligned}
& x(\varphi)=e \cdot \cos (\varphi) \\
& y(\varphi)=e \cdot \sin (\varphi)
\end{aligned}
$$

Based on this principle, a method for establishing the centre point curve of eccentrically rotating tools is presented in the following.
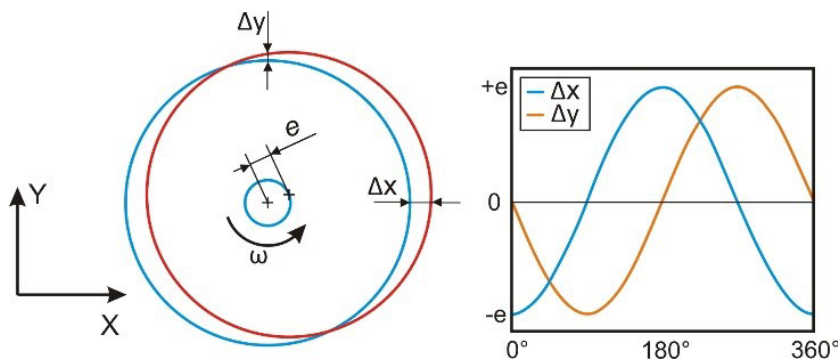

Fig. 1. Establishing the centre point curve of an eccentrically rotating circle 


\section{Method for measuring the eccentricity of the tool}

A suitable method for examining how unbalances influence the eccentricity of the tool axis in machine fixtures is presented in the following, based on the principle described in Chapter 2. This method makes it possible to establish the eccentricity caused by unbalances of the tool by means of comparing two distance signals, one in the case of a slow rotation of the tool and one at operating speed. Figure 2 represents schematically a test tool rotating around the fixed coordinate system at the angular velocity $\omega$. A sensor in the direction of the $\mathrm{X}$-axis here measures the surface profile of the tool during rotation.

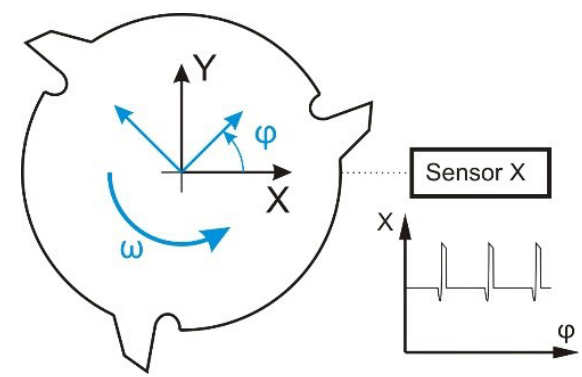

Fig. 2. Schematic test set-up for recording the surface profile of the tool

When the tool rotates slowly, it is hardly affected by centrifugal and unbalance forces. Thus, it can be assumed that the recorded surface profile in the area of the tool cutting edges is purely the tool geometry there. When the tool rotates at a higher rotational speed, unbalances lead to a displacement of the tool axis away from the axis of rotation, as described in Chapter 2. In order to establish the eccentricity of the tool caused in this way, a method for determining the tool eccentricity at operating conditions is described in the following, based on the comparison of the geometrical tool profile at $n \approx 0 \mathrm{~min}^{-1}$ and the superimposed profile at operating speed.

Figure 3 represents schematically the procedure for the data processing in the form of a block diagram. These data are processed with the algorithm described in the following, starting from the discrete signals in the two recorded measuring signals at $n \approx 0 \mathrm{~min}^{-1}$ and at operating speed. In order to process the signal proportions per tool revolution, striking topographies of the tool like teeth or chip spaces are used in the first step to clearly identify the angular position of the tool. The tooth flanks are identified in the algorithm for this, if a previously defined threshold is exceeded. Based on this, the signals measured can subsequently be saved in blocks, for example, per tool revolution. In the third step, the signals are synchronised by using striking profile areas in order to guarantee the phase balance of the tool profiles at slow and fast tool rotations. If the sampling frequency of the measuring device is not adjusted to the rotational speed in this method, the tool profile is scanned much more precisely at a low rotational speed than at the higher operating speed. In the fourth step, the signal at operating speed is interpolated to the same length as the signal at a low rotational speed in order to set off the signal proportions against each other. Then the signal blocks are put together again in the fifth step. The signals produced in this way can then be set off against each other so that a vibration signal caused by a tool unbalance can be calculated in the sixth step of the algorithm. The difference between the signals at operating speed and at a slow tool rotation is calculated for this. In the last step, the vibration signal is processed by filtering, and the frequency proportions in the signal are assessed with a fast Fourier transformation (FFT). 


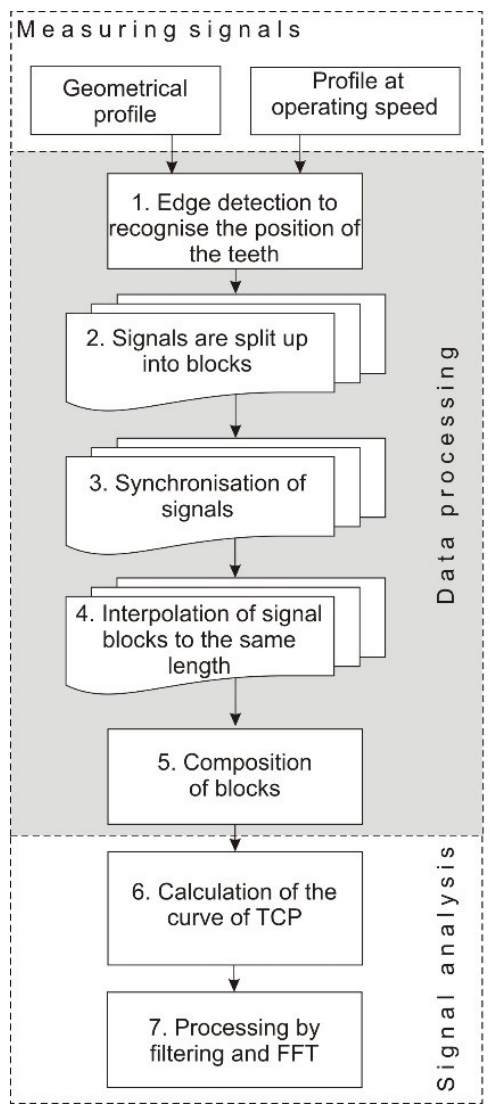

Fig. 3. Algorithm for signal processing in Matlab

\section{Measuring the eccentricity of the test tool}

The method described in Chapter 3 was tested with an inserted-tooth cutter by Leitz on a machining centre, type MAKA PE 170. A static unbalance of $U_{\text {mess }}=355 \mathrm{gmm}$ was specifically generated in the test tool with a mass of $m=5.9 \mathrm{~kg}$ by means of inserting balancing screws and then measured with a balancing machine by Haimer Tool Dynamic.

Figure 4 shows the measuring set-up for recording the surface profile of the tool during rotation. The surface profile of the tool was measured both in the X-direction and the Ydirection of the machine axes by using two laser triangulation sensors, type Keyence LKG32, with a sampling frequency of $f=50 \mathrm{kHz}$.

The profile recorded by the sensor was then converted into an analogue voltage signal and transferred to the signal analyser by Dactron, with which the information about the distance between tool and laser was saved in the ASCII format and afterwards evaluated with Matlab. 


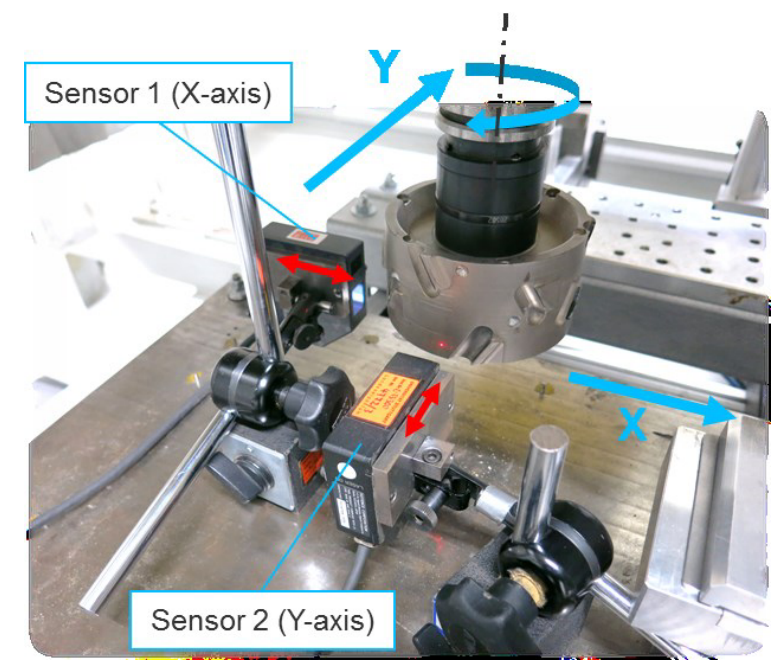

Fig. 4. Measuring set-up for establishing the profile of the test tool

The dynamic behaviour of the test tool was analysed with the method described in Chapter 3. The geometrical tool profile was recorded here for a rotational speed of $n=100 \mathrm{~min}^{-1}$. Afterwards the rotational speed was increased from $n=1,000 \mathrm{~min}^{-1}$ up to $n=5,000 \mathrm{~min}^{-1}$ in steps of thousands in order to demonstrate the influence of varying unbalance forces. As an example, Figure 5 on the top compares the signal of the geometrical profile with that of the measured profile for one revolution of the tool. The great amplitudes of the signal here represent the chip space and subsequently the tooth. A qualitative comparison of the measured tool profiles already showed that tooth 3 has a greater centre point path radius due to the unbalance. Using the evaluation procedure described in Chapter 3, it was then possible to synchronise the signals, process them and calculate the difference between the profiles. Figure 5 at the bottom illustrates the vibration profile of the tool calculated in this way. A clear sinusoidal vibration, superimposed with high-frequency signal proportions, can be seen here.

The protruding peaks occurred periodically per tooth and could be attributed to synchronisation errors of the two signals due to the algorithm described and measuring errors owing to the measuring method based on triangulation. The sensors used in the measurement had a measuring range of $\pm 5 \mathrm{~mm}$. As there are, however, greater differences in height at the tool owing to the cutting edges and the chip spaces, the measuring instrument got into an overload in the areas of the chip spaces. Upon the transition from overload back to the measuring range, there was an overshoot of the signal [10].

A Fourier transformation of the signals in the frequency range clearly showed a vibration frequency of $f=83.3 \mathrm{~Hz}$, which corresponds to the rotational frequency of the tool at operating speed. The vibration amplitude for $n=5,000 \mathrm{~min}^{-1}$ here corresponded to an eccentricity of the tool of $e=140 \mu \mathrm{m}$. This corresponded to the displacement of the tool caused by the unbalance and could be seen as unbalance-related deviation of the shaft axis from the axis of rotation.

In figure 6 , the curves of the TCP established in this way are shown in two directions for the signals recorded with the measuring set-up presented in Figure 5. The evaluated amplitudes of the first order showed that the deflection of the TCP in the X-direction of the machine was greater than in the Y-direction for all measurements. Hence, it could be assumed here that the TCP had an elliptical curve due to the anisotropy of the machine rigidity. 

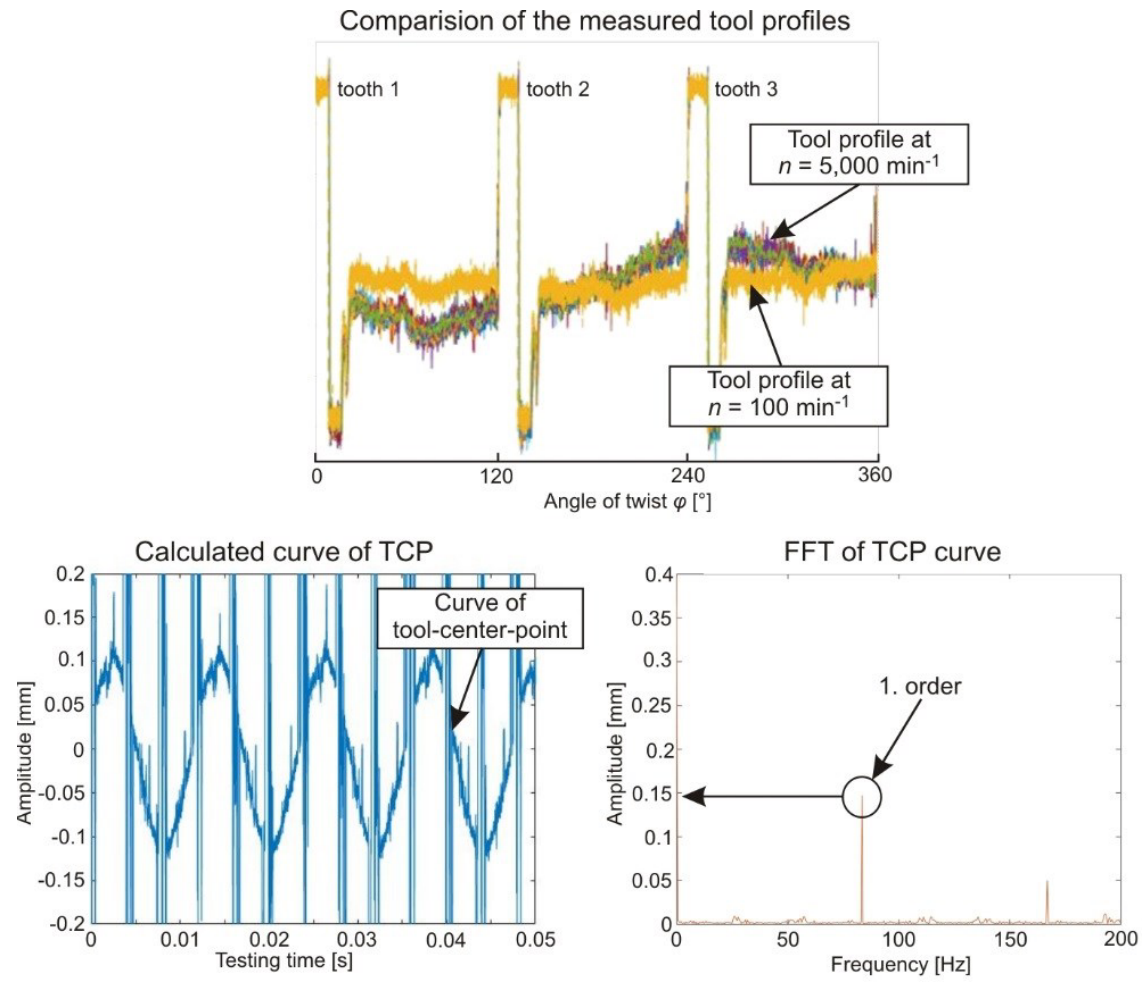

Fig. 5. Evaluation of the signals in sensor 2 at $n=5,000 \mathrm{~min}^{-1}$

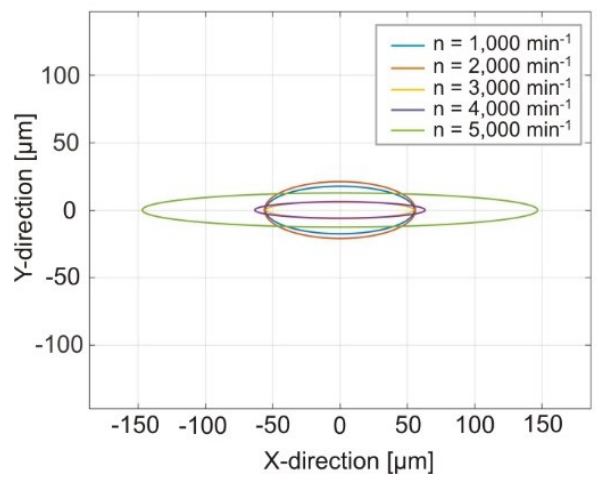

Fig. 6. Curves of the TCP at varying rotational speed

The eccentricity of the TCP could be measured with the method presented here. When comparing the curves of the TCP in Figure 6, it had to be taken into consideration that the unbalance forces increase quadratically with growing rotational speed. In this way, the measured eccentricity can be taken into account, for example, when calculating the waviness height or the path length of the tool's cutting edges to be expected. Figure 7 presents the flexibilities of the machine calculated from the ratio between the measured displacements of the TCP and the unbalance force of $U_{\text {mess }}=355 \mathrm{gmm}$ measured on the balancing machine. A relevant influence of the rotational frequency on the machine behaviour could absolutely be detected here. Regarding the analysed rotational speeds, a minimum of the flexibilities in both directions of space could be found at a rotational speed of $n=4,000 \mathrm{~min}^{-1}$. However, in view of arising unbalances it can absolutely be 
recommended to operate the tool at a rotational speed of $n=4,000 \mathrm{~min}^{-1}$ as the machine flexibility is expected to be lowest here. Since the tool also has a relatively great unbalance, the eccentricities are probably considerably lower in practice for a balanced tool as well.

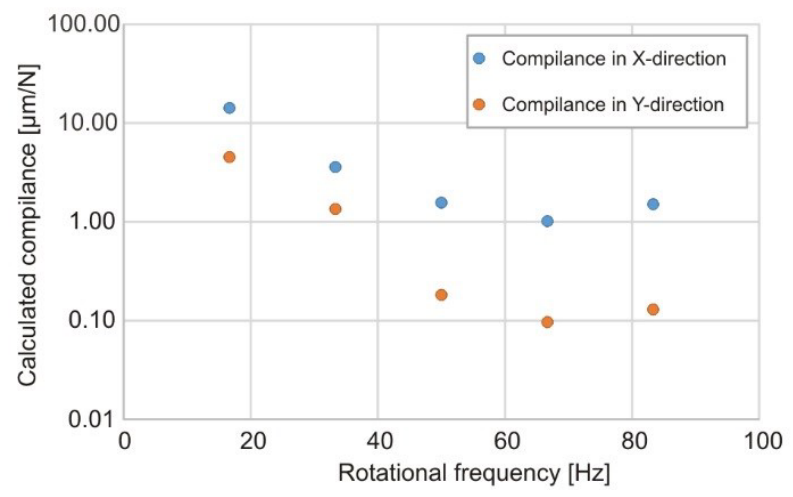

Fig. 7. Calculated flexibilities of the machine depending on rotational speed

\section{Conclusions}

In this paper, a method is presented for experimentally establishing the eccentricities of a tool caused by unbalances. It is based on comparing a measured tool profile at a low rotational speed and at the operating speed of the tool. It could be shown that unbalance-related displacements of the TCP can be reliably established with this method, which is applied to a 5-axis machining centre as an example. It was detected here that the tool showed a directionally dependent vibration behaviour, which could be attributed to different rigidities of the machine structure and had a direct influence on the eccentricities of the TCP. In addition, it could be shown that this method can be used to analyse the influence of the dynamic machine rigidity on the expected displacements of the tool. By comparing different designs of machines, it will be possible in this way to make recommendations on the permissible unbalance, based on the expected eccentricities of the tool.

\section{References}

1. H. Schneider: Auswuchttechnik, Springer-Vieweg, Berlin, Heidelberg, 8 (2013)

2. H. Schulz, T. Wuerz, CIRP Annals-Manuf. Techn.47.1, 321-324 (1998)

3. U. Heisel, A.Feinauer, Annals of CIRP 48.1, 321-324 (1999)

4. U. Heisel, H.Krondorfer, Proceed.of the 13th Internat. Wood Machining Seminar, Vancouver, 253-264, (1997)

5. U. Heisel, J. Tröger; S.Martynenko, Symp. on Wood Machining, Properties of Wood and Wood Composites Related to Wood Machining, Vienna, Austria, 161-173, (2004)

6. DIN 69888, Auswuchtanforderungen an rotierende Werkzeugsysteme, (Beuth-Verlag, Berlin, 2008)

7. ISO 15641, Milling cutters for high speed machining - Safety requirements, (Beuth-Verlag, Berlin, 2001)

8. DIN EN 847-1, Tools for woodworking - Safety requirements - Part 1: Milling tools, circular saw blades, (Beuth-Verlag, Berlin, 2013)

9. ISO 1940-1, Mechanical vibration - Balance quality requirements for rotors in a constant (rigid) state - Part 1: Specification and verification of balance tolerances, (Beuth-Verlag, Berlin, 2004)

10. K. C., Fan, Computer integrated Manuf. Syst., 10.4, 277-285 (1997) 